Draft Version September 7, 2018

Preprint typeset using $\mathrm{LAT}_{\mathrm{E}} \mathrm{X}$ style emulateapj v. 5/2/11

\title{
MAGNETIC FIELD AMPLIFICATION ASSOCIATED WITH THE RICHTMYER-MESHKOV INSTABILITY
}

\author{
Takayoshi Sano ${ }^{1}$, Katsunobu Nishinara ${ }^{1}$, Chiniro Matsuoka ${ }^{2}$, and Tsuyoshi Inoue ${ }^{3}$ \\ Draft version September 7, 2018
}

\begin{abstract}
The amplification of a magnetic field due to the Richtmyer-Meshkov instability (RMI) is investigated by two-dimensional MHD simulations. Single-mode analysis is adopted to reveal definite relation between the nonlinear evolution of RMI and the field enhancement. It is found that an ambient magnetic field is stretched by fluid motions associated with the RMI, and the strength is amplified significantly by more than two orders of magnitude. The saturation level of the field is determined by a balance between the amplified magnetic pressure and the thermal pressure after shock passage. This effective amplification can be achieved in a wide range of the conditions for the RMI such as the Mach number of an incident shock and the density ratio at a contact discontinuity. The results suggest that the RMI could be a robust mechanism of the amplification of interstellar magnetic fields and cause the origin of localized strong fields observed at the shock of supernova remnants.
\end{abstract}

Subject headings: instabilities — MHD — magnetic fields — shock waves — turbulence

\section{INTRODUCTION}

The Richtmyer-Meshkov instability (RMI) is of crucial importance in a variety of applications including astrophysical phenomena and laboratory experiments (Brouillette 2002; Nishihara et al. 2010). The RMI occurs when an incident shock strikes a corrugated contact discontinuity separating two fluids with different densities (Richtmver 1960; Meshkov 1969). Because of the corrugation of the interface, the surface profiles of the transmitted and reflected shocks are also rippled. The RMI is driven by the vorticity left by these rippled shocks at the interface and in the fluids (Wouchuk \& Nishihara 1997).

Supernova remnants (SNRs) are expected as a site of magnetic field amplification. Thin shell structure of the front shock in young SNRs observed by hard X-rays (e.g., Long et al. 2003; Bamba et al. 2003) requires the existence of strong fields with $\sim 100 \mu \mathrm{G}$, which is estimated from the syncrotron cooling length (Berezhko et al. 2003; Völk et al. 2005). Recent discovery of the year-scale variability in the synchrotron X-ray emission at the downstream regions of supernova shocks suggests that the magnetic field should be amplified up to the level of milligauss there (Uchivama et al. 2007; Uchivama \& Aharonian 2008).

In this paper, we consider the origin of this localized milligauss field in SNRs. Since the typical magnetic field in the interstellar medium (ISM) is of the order of microgauss (Beck 2001; Heiles \& Troland 2005), the amplification beyond simple shock compression is necessary to achieve the milligauss-order magnetic field. It is reported that observed synchrotron images could be modeled by random magnetic fields (Bvkov et al. 2008), but the amplification mechanism of the field is still an open question. As discussed in Uchivama et al. (2007), the pres-

\footnotetext{
sano@ile.osaka-u.ac.jp

${ }^{1}$ Institute of Laser Engineering, Osaka University, Suita, Osaka 565-0871, Japan

2 Department of Physics, Ehime University, Matsuyama, Ehime 790-8577, Japan

3 Department of Physics and Mathematics, Aoyama Gakuin University, Sagamihara, Kanagawa 252-5258, Japan
}

ence of milligauss magnetic field has a critical meaning in the long-standing paradigm of cosmic-ray proton acceleration in young SNRs (Bell 1978; Blandford \& Ostriker 1978). The investigation on the physical origin of such strong field is then an inevitable step to clarify the role of the milligauss field.

It is widely known that the ISM has nonuniform density structures of warm and cold neutral media formed by the thermal instability (Field et al. 1969; Kovama \& Inutsuka 2002; Inoue et al. 2009). The electron density fluctuation in hot ionized medium is also observed as an evidence of the interstellar turbulence (Armstrong et al. 1995). The RMI can take place when supernova shocks pass through these inhomogeneous materials, and which is a quite common event in the ISM. The low- and mid-temperature parts of the ISM is partially ionized by cosmic rays. Although the ionization fraction is very small, it is well described by the ideal MHD approximation. Hence the interstellar magnetic fields could be affected by the fluid motions of the RMI.

Turbulence and magnetic field amplification has been studied for shock wave propagation through the inhomogeneous ISM (Giacalone \& Jokipii 2007; Inoue et al. 2009, 2012; Guo et al. 2012). Field amplification is inferred to take place both in the star's collapsing core (Endeve et al. 2010) and in the progenitor wind overrun by the shock (Chevalier 1992), and in both cases the shock itself has been suggested as the cause of the amplification. Inoue et al. (2012) examined the three-dimensional magnetohydrodynamical interaction between clumpy interstellar clouds formed by the thermal instability and a strong shock wave of young SNRs with the typical velocity $\sim 3000 \mathrm{~km} \mathrm{~s}^{-1}$. It is found that the maximum strength of the interstellar field increases rapidly owing to the shear layer at the cloud interface, in addition to rather gradual growth of the average field strength by the so-called turbulent dynamo (or turbulent stretching of magnetic field). The maximum field strength reaches $1 \mathrm{mG}$ in their simulations, which is larger by an order than the volume averaged strength. The strong-field regions are localized in thin layers at the 
interface.

Turbulent dynamo is one of the promising effects to amplify the magnetic field in the postshocked regions. This process dominates the growth of the volume averaged field strength, which has been recognized by many authors (Giacalone \& Jokipii 2007; Mizuno et al. 2011). On the other hand, the size of the strong-field regions inferred from X-ray observations is typically $0.05 \mathrm{pc}$ (Uchivama et al. 2007), and this would be much smaller than the preshock inhomogeneity scale $\sim 1$ pc. Such localized strong field should be attributed to the shockcloud interaction, and characterized by the maximum strength rather than the average. Therefore, the evolution of the maximum field strength is a key to understand the formation of the localized milligauss regions. In this paper, we demonstrate the RMI could be an efficient mechanism of the field amplification at the cloud interface, which can enhance the maximum strength locally by a factor of more than 100 .

Shock-cloud interaction with a magnetic field has been studied numerically by multi-dimensional simulations (e.g., van Loo et al. 2007; Shin et al. 2008). In their works, the time evolutions of the entire region of a roundshaped cloud are considered. In contrast, we focus on the hydrodynamical instability arising from the spacial fluctuations at the cloud surface aiming to see much smaller scale structures. The advantage of this approach is that we can make use of the quantitative knowledge of RMI for the interpretation of the results.

MHD effects on the RMI are investigated firstly in the context of the dynamics of the magnetosphere (Wu \& Roberts 1999). Samtanev (2003) has demonstrated by numerical simulations that the growth of the RMI is suppressed in the presence of a magnetic field. Depending on the field direction relative to the incident shock surface, the magnetic field works differently on the fluid motions of RMI. When the magnetic field is aligned with the motion of the shock, the suppression of the instability is caused by changes in the shock refraction process at the contact discontinuity. The role of the magnetic field is to prevent the deposition of circulation on the interface (Wheatley et al. 2005, 2009). When the magnetic field is parallel to the shock surface, the instability is stabilized by the Lorentz force (Cao et al. 2008).

Previous works on the MHD RMI is mostly focused on the suppression effects by a strong magnetic field. Here, we concentrate our discussions on the evolution of an ambient magnetic field affected by the nonlinear motions of the RMI. The velocity of the supernova shocks is typically $100-1000 \mathrm{~km} \mathrm{~s}^{-1}$, and then the Mach number can be 10-100 in the warm diffuse gas. The thermal instability produces the density gap between the cold and warm neutral media and the ratio is about 10-100. Thus, the RMI associated with the supernova shocks is characterized by a large Mach number and a large density ratio. In this paper, we show the results of MHD simulations performed with a variety of initial conditions including such extreme cases.

Single-mode analysis of the RMI is adopted in order to examine the field evolution and understand the physical mechanism. The plan of this paper is as follows. In $\S 2$, the basic equations, initial conditions, and numerical method are described. In this section, we make a brief review on the characteristics of the RMI, which is useful for the interpretation of our results. Numerical outcomes of two-dimensional MHD simulations are shown in $\S 3$. The time evolutions of the field for various initial conditions are examined in this section. In $\S 4$, the saturation mechanism of the amplified field and parameter dependence of the results are discussed. Finally, our conclusions are summarized in $\S 5$.

\section{METHOD}

\subsection{Basic Equations and Initial Conditions}

To study the nonlinear evolutions of the RMI, the equations of ideal MHD are solved;

$$
\begin{gathered}
\frac{\partial \rho}{\partial t}+\nabla \cdot(\rho \boldsymbol{v})=0, \\
\frac{\partial \rho \boldsymbol{v}}{\partial t}+\nabla \cdot\left[\left(P+\frac{B^{2}}{8 \pi}\right) \boldsymbol{I}+\rho \boldsymbol{v} \boldsymbol{v}-\frac{\boldsymbol{B} \boldsymbol{B}}{4 \pi}\right]=0, \\
\frac{\partial e}{\partial t}+\nabla \cdot\left[\left(e+P+\frac{B^{2}}{8 \pi}\right) \boldsymbol{v}-\frac{(\boldsymbol{B} \cdot \boldsymbol{v}) \boldsymbol{B}}{4 \pi}\right]=0, \\
\frac{\partial \boldsymbol{B}}{\partial t}=\boldsymbol{\nabla} \times(\boldsymbol{v} \times \boldsymbol{B}),
\end{gathered}
$$

where $\rho, \boldsymbol{v}$, and $\boldsymbol{B}$ are the mass density, velocity, and magnetic field, respectively, and $e$ is the total energy density,

$$
e=\frac{P}{\gamma-1}+\frac{\rho v^{2}}{2}+\frac{B^{2}}{8 \pi} .
$$

In our simulations, the equation of state for ideal gas is adopted with the adiabatic index $\gamma=5 / 3$.

The initial configuration for our single-mode analysis is illustrated in Figure 1 a. Two fluids with different densities, $\rho_{1}$ and $\rho_{2}\left(>\rho_{1}\right)$, are separated by a contact discontinuity at $y=Y_{\mathrm{cd}}$. A shock propagating through the light fluid " 1 " $\left(y>Y_{\mathrm{cd}}\right)$ hits the interface at a time $t=0$. The incident shock velocity is $U_{i}(<0)$ and the fluid velocity behind the shock is $V_{1}(<0)$. Let $P_{0}$ be a uniform pressure of the both fluids before shock passage. The Mach number of the incident shock is defined as $M=\left|U_{i}\right| / c_{s 1}$ where $c_{s 1}=\left(\gamma P_{0} / \rho_{1}\right)^{1 / 2}$ is the sound speed of the fluid " 1 ". Here the $x$ - and $y$-axis are set to be perpendicular and parallel to the shock surface.

The interface has an initial corrugation of a sinusoidal form, $y=Y_{\mathrm{cd}}+\psi_{0} \cos (k x)$, where $\psi_{0}$ is a corrugation amplitude, $k=2 \pi / \lambda$ is the perturbation wavenumber, and $\lambda$ is the wavelength. This spatial corrugation of the interface is an essential ingredient for the occurrence of the RMI.

The initial geometry of a magnetic field is assumed to be uniform with the size of $|\boldsymbol{B}|=B_{0}$ in the preshocked region $\left(y<Y_{\text {is }}\right)$. As for the field direction, three cases are considered and those are for the cases of perpendicular MHD shocks $\left(B_{x}=B_{0}\right)$, parallel shocks $\left(B_{y}=B_{0}\right)$, and oblique shocks $\left(B_{x}=B_{y}\right)$. The physical quantities in the postshocked region behind the incident shock are calculated from the Rankine-Hugoniot conditions for MHD shocks.

The initial configuration depicted by Figure 1 $a$ can be characterized by only four non-dimensional parameters. The sonic Mach number $M$ parameterizes the incident shock velocity. The contact discontinuity is expressed 
by the density jump $\rho_{2} / \rho_{1}$ and the ratio of the corrugation amplitude to the wavelength $\psi_{0} / \lambda$. The initial field strength is given by the plasma beta $\beta_{0}=8 \pi P_{0} / B_{0}^{2}$ which is the ratio between the gas and magnetic pressures defined at the preshocked region. Thanks to the simplicity of the system, a variety of situations can be examined only by choosing these four parameters; $M$, $\rho_{2} / \rho_{1}, \psi_{0} / \lambda$, and $\beta_{0}$.

\subsection{Characteristic Scales for the RMI}

Figure $1 b$ is a schematic picture around the contact discontinuity after the shock passage. Now, the contact discontinuity moves with the velocity $v^{*}$. The pressure $P^{*}$ becomes higher compared to the initial $P_{0}$. The incident shock is transmitted into the heavy fluid " 2 " $\left(y<Y_{\mathrm{cd}}\right)$ which moves with the velocity $U_{t}(<0)$, and the density behind the transmitted shock is $\rho_{2}^{*}$. Similarly, a reflected shock forms with the velocity $U_{r}(>0)$ and compresses the fluid " 1 " to the density $\rho_{1}^{*}$. If the interface is corrugated, the shock fronts of these two waves are also rippled. Then, refraction of the fluids across the shocks occurs as indicated by the arrows in Figure 10.

Consider fluid motions near the shock surface in a frame moving with the transmitted or reflected shock. For the both shocks, the upstream fluid moves along the $y$-axis. However, the downstream motion should have the tangential component as a consequence of the obliqueness of the shock front. When the corrugation amplitude is small, the velocities of these tangential flows are given by

$$
\begin{gathered}
\delta v_{1}^{*}=k \psi_{r}\left(v^{*}-V_{1}\right), \\
\delta v_{2}^{*}=k \psi_{t} v^{*}
\end{gathered}
$$

(Wouchuk \& Nishihara 1997), where $\psi_{r}=\psi_{0}\left(1-U_{r} / U_{i}\right)$ and $\psi_{t}=\psi_{0}\left(1-U_{t} / U_{i}\right)$ are the initial ripple amplitudes for the reflected and transmitted shocks. The tangential shear motion across the contact discontinuity generates the vorticity at the interface, and which is the driving source of unstable growth for the RMI.

In the RMI, the shock acceleration is impulsive and causes the perturbation amplitude to grow linearly in time. Richtmyer (1960) has proposed a simple estimate of the growth rate of the amplitude; $\partial \psi / \partial t=k \psi_{0}^{*} A^{*} v^{*}$ where $\psi_{0}^{*}=\psi_{0}\left(1-v^{*} / U_{i}\right)$ is the interface amplitude just after shock passage and $A^{*}=\left(\rho_{1}^{*}-\rho_{2}^{*}\right) /\left(\rho_{1}^{*}+\rho_{2}^{*}\right)$ is the Atwood number of the postshocked interface. This prescription is obtained by a generalization of the RayleighTaylor formula with an impulsive acceleration.

More complex analytic formulas for the asymptotic growth rate of RMI are derived by Wouchuk \& Nishihara (1996, 1997). In the weak shock limit, the linear growth velocity $v_{\text {lin }}$ can be written using the tangential velocities $\delta v_{1}^{*}$ and $\delta v_{2}^{*}$ as

$$
v_{\text {lin }}=\frac{\rho_{1}^{*} \delta v_{1}^{*}-\rho_{2}^{*} \delta v_{2}^{*}}{\rho_{1}^{*}+\rho_{2}^{*}},
$$

and then the timescale of the RMI is characterized by

$$
t_{\mathrm{rm}}=\frac{1}{k v_{\text {lin }}} .
$$

Within the linear regime, the spike and bubble grow with the same velocity $v_{\text {lin }}$. For the weak shock cases, it is sufficient that the vorticity only at the interface is considered for the derivation of $v_{\text {lin }}$. However, for the strong shock cases, the bulk vorticity left behind the rippled transmitted shock cannot be ignored. It is known that the bulk vorticity reduces the growth of the RMI. Therefore, in the strong shock limit, the growth velocity given by Equation (8) will be overestimate by a factor of a few (Wouchuk \& Nishihara 1997). Furthermore the growth rate of the RMI could be reduced in the presence of a magnetic field (Wheatley et al. 2005, 2009). However, for sake of simplicity, we used Equations (8) and (9) as the typical scales of the RMI throughout our analysis in this paper.

Once the initial conditions are set, the growth velocity $v_{\text {lin }}$ can be derived by solving an appropriate MHD Riemann problem. The analytic formula given by Equation (8) intimates that the faster growth can be realized by the larger amplitude $\psi_{0}$ and/or the larger Mach number $M$. When $M \gtrsim 2$, the growth velocity is roughly proportional to $M$. This means that the growth time of the RMI is quite different depending on the incident shock strength. In the limit of high density ratio, $\rho_{2} / \rho_{1} \gg 1$, the growth velocity is approximately estimated as $v_{\text {lin }} \sim k \psi_{0}\left|v^{*}\right| \sim k \psi_{0}\left(\rho_{2} / \rho_{1}\right)^{-1 / 2}\left|V_{1}\right|$. However, within the range of $\rho_{2} / \rho_{1} \sim 1.5-100$ considered in our analysis, the growth time $t_{\mathrm{rm}}$ has little dependence on the density jump.

Just for reference, we estimate the characteristic quantities related to the RMI occurring in the ISM. The shock velocity of SNRs is $100-1000 \mathrm{~km} \mathrm{~s}^{-1}$, so that the typical Mach number is $M \sim 10-100$ in the warm neutral medium. It is a common feature in the ISM that nonuniform structures consisted of warm and cold neutral media. The cold and dense filamentary clumps and the surrounding warm diffuse gas are formed as a natural consequence of the thermal instability (Field 1965; Kovama \& Inutsuka 2000). The density ratio between the two phases is about 10-100. The length of the filament $(\sim 1 \mathrm{pc})$ is roughly given by the most unstable scale of the thermal instability, and the thickness of the filament is about $0.1 \mathrm{pc}$ (Inoue et al. 2009). At the boundary between the two phases, there exists a transition layer, the size of which is determined by the socalled Field length $(\sim 0.01-0.1 \mathrm{pc})$. It is known that the transition layer is unstable under corrugational deformations (Inoue et al. 2006; Stone \& Zweibel 2009). The most unstable wavelength of this instability at the evaporation/condensation front is typically $0.1 \mathrm{pc}$ and the growth time is approximately equal to the cooling time. Then we can assume the initial corrugation amplitude for the RMI is of the order of the ratio of the transition thickness to the most unstable wavelength, i.e., $\psi_{0} / \lambda \sim 0.1$.

Using these typical quantities of the ISM, the growth velocity is estimated as $v_{\operatorname{lin}} / c_{s 1} \sim 1-10$ and the timescale of RMI is roughly given by $t_{\mathrm{rm}} / t_{s 1} \sim 0.1-0.01$, where $c_{s 1}$ is the sound speed in the diffuse gas and $t_{s 1}=\lambda / c_{s 1}$ is the sound crossing time. Assuming $c_{s 1} \sim 10 \mathrm{~km} \mathrm{~s}^{-1}$ and $\lambda \sim 0.1 \mathrm{pc}$, these values are corresponding to $v_{\text {lin }} \sim$ $10-100 \mathrm{~km} \mathrm{~s}^{-1}$ and $t_{\mathrm{rm}} \sim 10^{2}-10^{3} \mathrm{yr}$. Then the growth time $t_{\mathrm{rm}}$ is much shorter than the cooling time $t_{\text {cool }}$ which is a few Myr in the typical ISM (Inoue et al. 2009). In the analysis below, we choose $M=10, \rho_{2} / \rho_{1}=10$, and $\psi_{0} / \lambda=0.1$ as the parameters for a fiducial case. 


\subsection{Numerical Scheme and Grid}

We solve the ideal MHD equations by using a conservative Godunov-type scheme (e.g., Sano et al. 1998). Operator splitting algorithm is adopted in our scheme. The hydrodynamical part of the equations is solved by a second-order Godunov method, using the exact solutions of a simplified MHD Riemann problem. The one-dimensional Riemann solver is simplified by including only the tangential component of a magnetic field. The characteristic velocity is then the magnetosonic wave alone, and then the MHD Riemann problem can be solved in a way similar to the hydrodynamical one (Colella \& Woodward 1984). The piecewise linear distributions of flow quantities are calculated with a monotonicity constraint following van Leer's method (van Leer 1979). The remaining terms, the induction equation and the magnetic tension part of the equation of motion, are solved by the consistent MoC-CT method (Stone \& Norman 1992; Clarke 1996), guaranteeing $\nabla \cdot \boldsymbol{B}=0$ within round-off error throughout the calculation (Evans \& Hawlev 1988).

The numerical scheme includes an additional numerical diffusion in the direction tangential to the shock surface in order to care the carbuncle instability (Hanawa et al. 2008). The numerical viscosity is added only in the regions where the characteristics of either fast or slow wave converges, i.e., in the regions potentially dangerous to the carbuncle instability. This treatment could be crucial in our analysis because this numerical instability influences the size of baroclinic vorticity, which is generated by the misalignment of the pressure gradient of the shock and the local density gradient across the interface.

Calculations are carried out in a frame moving with the velocity $v^{*}$ which is the interface velocity after the interaction with the incident shock. For convenience, we define the locus of $y=0$ as where the incident shock reaches the contact discontinuity at $t=0$. Then the postshocked interface stays at $y=0$ if it is not corrugated initially, or stable for the RMI. The simulations start before the incident shock hits the corrugated interface $\left(\left|Y_{\text {is }}-Y_{\text {cd }}\right|>\psi_{0}\right)$, and thus the initial time is negative $t_{0}=-\left|Y_{\text {is }}-Y_{\mathrm{cd}}\right| /\left|U_{i}\right|$.

The system of equations is normalized by the initial density and sound speed of the light fluid " 1 ", $\rho_{1}=1$ and $c_{s 1}=1$, and the wavelength of the density fluctuation $\lambda=1$. The sound crossing time is also unity in this unit, $t_{s 1}=\lambda / c_{s 1}=1$. Most of the calculations in this paper use a standard resolution of $\Delta_{x}=\Delta_{y}=\lambda / 256$ unless otherwise stated. A periodic boundary condition is used in the $x$-direction, and an outflow boundary condition is adopted in the $y$-direction. The size of the computational box in the $x$-direction is always set to be $L_{x}=\lambda$. The $y$-length, on the other hand, is taken to be relatively larger, $L_{y} \geq 10 \lambda$. The choice of $L_{y}$ is depending on the initial model parameters. All the calculations are stopped before the transmitted shock reaches the edge of the computational domain.

\section{RESULTS}

\subsection{Magnetic Field Amplification}

Single-mode analysis of the RMI and the amplification of a magnetic field are investigated by two-dimensional MHD simulations. Figure 2 shows the two-dimensional images of the density and field strength at the nonlinear regime of RMI. The initial parameters of this fiducial model are the Mach number $M=10$, the density jump $\rho_{2} / \rho_{1}=10$, and the corrugation amplitude $\psi_{0} / \lambda=0.1$. A uniform magnetic field parallel to the shock surface, $\left(B_{x}, B_{y}\right)=\left(B_{0}, 0\right)$, is assumed initially, and thus this case is for a perpendicular MHD shock.

In order to elucidate passive evolution of the field, the initial strength is supposed to be very weak, $\beta_{0}=10^{8}$ at the upstream of the incident shock. Even though this weak field does not affect the dynamics of the RMI, the spatial distribution of the field can be modified dramatically by the fluid motions driven by the RMI. Hereafter, we use the time normalized by the timescale of $\mathrm{RMI}, t_{\mathrm{rm}}=\left(k v_{\mathrm{lin}}\right)^{-1}$. The ratio between $t_{\mathrm{rm}}$ and the sound crossing time $t_{s 1}$ depends on the model parameters. Most of the cases, $t_{\mathrm{rm}}$ is shorter than $t_{s 1}$. For example, $t_{\mathrm{rm}}=0.116$ for the fiducial model, while $t_{s 1}$ is always unity in our simulations. The snapshot data shown by Figure 2 are taken at the normalized time $k v_{\text {lin }} t=10$.

The density profile shown in Figure $2 a$ exhibits a mushroom-shaped spike as a result of the growth of RMI. This figure is a close-up view focused around the contact discontinuity at $y=0$. The loci of the transmitted and reflected shocks at this time are further out of this figure, that is, $y=-1.6 \lambda$ and $5.6 \lambda$, respectively. The spike height reaches $y \sim 0.7 \lambda$, which is 7 times larger than the initial amplitude $\psi_{0}$. In Figure $3 a$, the time history of the distance from the spike top to the bubble bottom $d_{s b} \equiv\left|y_{s}-y_{b}\right|$ is shown as a function of the normalized time $k v_{\text {lin }} t$, where $y_{s}$ and $y_{b}$ are the $y$-coordinate of the spike and bubble.

Figure $3 a$ also shows the growth velocities of the RMI, which are evaluated by the advection velocities of the spike $v_{s}$ and bubble $v_{b}$. The growth velocity of the spike $v_{s}$ increases rapidly just after the incident shock hits the interface, and takes the maximum when $k v_{\text {lin }} t \sim 2$. The maximum is comparable to the analytic prediction $v_{\text {lin }}$ given by Equation (8). The growth velocity $v_{\text {lin }}$ is subsonic compared to the sound speed in the shocked regions, $v_{\text {lin }}=0.20 c_{s 1}^{*}=0.48 c_{s 2}^{*}$ where $c_{s 1}^{*}=\left(\gamma P^{*} / \rho_{1}^{*}\right)^{1 / 2}$ and $c_{s 2}^{*}=\left(\gamma P^{*} / \rho_{2}^{*}\right)^{1 / 2}$, but it is supersonic relative to the preshocked sound speed, $v_{\text {lin }}=1.37 c_{s 1}$. The spike continues to grow with the velocity about $v_{s} \sim 0.2 v_{\text {lin }}$ until at least $k v_{\text {lin }} t=30$ in this model.

The bubble growth, on the other hand, is quenched at the earlier phase of the evolution. This is a general feature of the RMI (Matsuoka et al. 2003), especially for the strong shock cases as examined in this paper. The suppression is caused by the bulk vorticity comes from the deformed transmitted shock. It is worth noticing that weak spike-like patterns can be seen in the density distribution behind the transmitted shock in the heavy fluid "2". This is also associated with the bulk vorticity generated by the propagation of a rippled shock (e.g., Ishizaki \& Nishihara 1997). As the shock separates away from the interface, their corrugation amplitude oscillates and decreases with time. The amplitude of the bulk vorticity is larger for the cases with higher shock strength, and it could affect the nonlinear behavior of the RMI (Wouchuk \& Nishihara 1997; Nishihara et al. 2010). Although the effects of the bulk vorticity might be important for the interstellar strong shocks, its quantitative 
details is beyond the scope of this paper.

Figure $2 b$ depicts the spatial distribution of the field strength. The magnetic field is already amplified more than 200 times at $k v_{\operatorname{lin}} t=10$. The strong field regions are localized at the mushroom cap and form thin filamentary structures along the interface. Figure $3 b$ shows the time evolution of the maximum field strength $|\boldsymbol{B}|_{\max }$ in the computational domain. Before the incident shock hits the corrugated interface, the $y$-component of the field is nothing in this model. The Mach number $M=10$ is large enough that the density behind the incident shock is enhanced by a factor of $(\gamma+1) /(\gamma-1) \approx 4$ due to shock compression. Then the initial $\left|B_{x}\right|$ max is larger by the same factor than $B_{0}$.

During the interaction between the incident shock and interface, $B_{y}$ appears near the rippled interface due to the refraction of the flow. The maximum value $\left|B_{y}\right|_{\max }$ grows linearly in time at the very beginning. Then each component of the field increases exponentially until $k v_{\text {lin }} t \sim 2$ during the growth velocity of the spike $v_{s}$ is comparable to $v_{\text {lin }}$ (see Figure $3 a$ ). At this stage, $\left|B_{x}\right|_{\max }$ is still dominant over $\left|B_{y}\right|_{\max }$. But the both components become comparable and evolve in a similar manner at the later evolutionary stage $k v_{\text {lin }} t \gtrsim 5$. The early growth rates $\sigma_{B}$ obtained by using a fitting formula,

$$
|\boldsymbol{B}|_{\max }(t) \propto \exp \left(\frac{\sigma_{B} t}{t_{\mathrm{rm}}}\right)
$$

are $\sigma_{B}=1.0$, which is shown by the dotted line in the figure. The maximum strength increases with time even at $k v_{\text {lin }} t=30$ and exceeds $|\boldsymbol{B}|_{\max } \sim 10^{3} B_{0}$ at the end. These results are obviously suggesting that the RMI is a quite efficient mechanism of magnetic field amplification.

\subsection{Physical Mechanism of the Amplification}

In this subsection, we consider the physical mechanism of the field amplification associated with the RMI. The induction equation for the ideal MHD can be rewritten as

$$
\frac{\partial \boldsymbol{B}}{\partial t}=-(\boldsymbol{v} \cdot \boldsymbol{\nabla}) \boldsymbol{B}+(\boldsymbol{B} \cdot \boldsymbol{\nabla}) \boldsymbol{v}-\boldsymbol{B}(\boldsymbol{\nabla} \cdot \boldsymbol{v}),
$$

where each term of the right-hand-side stands for advection, stretching, and compression. Among these terms, net increase of the field can be done only through the stretching and compression.

Figure $4 a$ shows the magnetic field lines in the neighborhood of the interface for the fiducial model at $k v_{\text {lin }} t=$ 2. The gray color denotes the higher density part in the fluid " 2 " bounded between the contact discontinuity and transmitted shock front. As mentioned above, the magnetic field is amplified efficiently at the interface between two fluids. The positions of the largest strength are shown by the crosses in this figure. The fluid motion excited by the RMI stretches the surface area of the contact discontinuity. It is found that the field amplification is predominantly originated from this stretching effect.

The relative importance of stretching and compression can be evaluated from our numerical results. Multiplying the magnetic field vector $\boldsymbol{B}$ to Equation (11), the following scalar equation can be derived;

$$
\frac{1}{2} \frac{\partial}{\partial t}|\boldsymbol{B}|^{2}=-\boldsymbol{B} \cdot(\boldsymbol{v} \cdot \boldsymbol{\nabla}) \boldsymbol{B}+\boldsymbol{B} \cdot(\boldsymbol{B} \cdot \boldsymbol{\nabla}) \boldsymbol{v}-|\boldsymbol{B}|^{2} \boldsymbol{\nabla} \cdot \boldsymbol{v}
$$

where the last two terms represent stretching and compression.

Using the snapshot data same as in Figure 4a, we calculate the sizes of the stretching and compression terms in Equation (12), which are shown in Figures 43 and 4 c, respectively. The same color contours are used in these two figures. Focusing on the mushroom-cap region, the stretching term takes the maximum, while the compression term is almost nothing there. Because the fluid motions in the RMI is mostly incompressible, so that the contribution of compression toward the field amplification is negligibly small compared to that of stretching. The compression term is larger at the transmitted shock front, but that is simply because of shock compression. The strong field regions along the interface perfectly match where the stretching term is dominant over the other terms. This is a clear evidence that the stretching effect is the major source of the field amplification.

To verify this interpretation, we compare quantitatively the stretching rate of the interface and the growth rate of the field. The stretching rate can be obtained numerically from a nonlinear vortex sheet model (Matsuoka et al. 2003). After the transmitted and reflected shocks have traveled a distance larger than the fluctuation wavelength $\lambda$, the system can be regarded as incompressible and irrotational except for the interface on which nonuniform vorticity is induced by the shocks. We treat the interface as a curve with the use of a Lagrangian parameter in the $x-y$ plane. The governing equations for the nonlinear analysis are the Bernoulli equation and kinematic boundary conditions.

The Bernoulli equation, i.e., the pressure continuous condition at the interface, is given by

$$
\rho_{1}^{*}\left[\frac{\partial \phi_{1}}{\partial t}+\frac{1}{2}\left(\nabla \phi_{1}\right)^{2}\right]=\rho_{2}^{*}\left[\frac{\partial \phi_{2}}{\partial t}+\frac{1}{2}\left(\nabla \phi_{2}\right)^{2}\right],
$$

where $\phi_{i}(i=1,2)$ is the velocity potential defined as $\nabla \phi_{i}=\boldsymbol{v}_{i}$ and $\boldsymbol{v}_{i}$ is the velocity of the fluid " $i$ ". In order to calculate the interfacial motion as a vortex sheet, we need to rewrite Equation (13) into an evolution equation for the vortex sheet strength $\kappa$. Here, $\kappa=|\kappa|$ is defined by the circulation $\Gamma \equiv \phi_{1}-\phi_{2}$ as $\boldsymbol{\kappa}=\nabla \Gamma$. The detailed derivation of the evolution equation associated with the vortex-induced velocity is explained in the appendix.

The obtained shape of the interface at $k v_{\operatorname{lin}} t=2$ is shown by Figure 4 . The line color indicates the stretching rate $\sigma_{\text {int }}$ at each Lagrangian point on the interface, which is defined as

$$
\sigma_{\text {int }} \equiv \frac{t_{\mathrm{rm}}}{l} \frac{d l}{d t}
$$

where $l$ is the line element along the interface. The vortex sheet result reproduces surprisingly well the spatial distribution of the stretching size in our simulation shown by Figure 43 . The large stretching rate can be seen at the spike top, and this feature coincides exactly with the strong field region as shown in Figure 4a. The vortex sheet model predicts that the largest stretching rate appears at the side of the mushroom cap in the later phase. This is also consistent with the MHD results at $k v_{\text {lin }} t=10$ depicted by Figure $2 b$. Furthermore the maximum stretching rate $\sigma_{\text {int }} \sim 0.8$ is fairly close to the 
growth rate of the magnetic field $\sigma_{B} \sim 1.0$. This is another fact to convince us of the strong relation between the field amplification and the stretching effect.

\subsection{Dependence on the Initial Field Geometry}

The orientation of interstellar magnetic fields has no correlation with the direction of supernova shocks. Therefore, it is interesting to examine the effects of the initial field direction on the amplification process. A parallel shock case with the initial field $\left(B_{x}, B_{y}\right)=$ $\left(0, B_{0}\right)$ and an oblique shock case with $\left(B_{x}, B_{y}\right)=$ $\left(B_{0} / \sqrt{2}, B_{0} / \sqrt{2}\right)$ are performed for this purpose. The other parameters are identical to the fiducial model, so that the magnetic field is again assumed to be very weak, $\beta_{0}=10^{8}$. The physical quantities in the downstream of the incident shock are calculated from the jump conditions for MHD shocks, and the fast shock condition is used for the oblique shock case.

The field amplification due to the RMI is found to be independent of the direction of the ambient field. Figure 5 shows the time evolutions of the maximum field strength for the models with different initial field geometries. First, let us take a look at the early stage of the evolution until $k v_{\operatorname{lin}} t \approx 10$. For all the models, the magnetic field increases exponentially at this stage and is amplified up to about 100 times as large as the initial strength $B_{0}$. The stretching term always contributes the field amplification dominantly. The difference in the maximum strength at $t=0$ is caused by the compression of the tangential field $B_{x}$ behind the incident shock. For the strong shock limit, the ratio of the initial $\left|B_{x}\right|_{\max }$ in our models should be (parallel) : (oblique) : (perpendicular) $=1: 2 \sqrt{2}: 4$. The highest field at $k v_{\text {lin }} t=10$ is achieved in the perpendicular shock case, but this is mainly because of the difference in the initial $|\boldsymbol{B}|_{\max }$. The amplification factor from the initial maximum field is rather similar for all these models. The reason is that the evolution of the RMI is unaffected by the weak field, and then the stretching motion is almost identical no matter which direction the ambient field takes.

Figures $6 a$ and $6 b$ show the spatial profile of the field strength and the field lines, respectively, for the oblique shock case. These snapshots are taken at $k v_{\text {lin }} t=10$ the same as Figure 2. The density distribution is almost identical to that in the fiducial model shown by Figure 2a. Although asymmetric structures can be seen in the amplified field because of the shock obliqueness, the strong-field regions are concentrated along the interface at the mushroom-shaped spike. The maximum field appears near the top of the spike and the strength is $|\boldsymbol{B}|_{\max } / B_{0}=192$ at this time. This result strongly supports that the field amplification in the oblique shock case is also caused by the surface stretching of the contact discontinuity as well as in the perpendicular shock case (fiducial model).

At the later evolutionary stage $10 \lesssim k v_{\text {lin }} t \lesssim 30$, the maximum $|\boldsymbol{B}|$ keeps increasing with time in the perpendicular and oblique shock cases (see Fig. 51). When the fluid " 1 " has a tangential field component, $B_{x}$, the total magnetic flux swept by the spike increases as long as the growth of RMI continues. On the other hand, if there is only a normal component, $B_{y}$, the magnetic flux involved by the RMI is fixed by the initial setting. Then the size of the maximum field have to be saturated when all the flux is confined in a sufficiently small region. This is why the parallel shock case shows the saturation of the growth, a flat plateau in the time-profile of $|\boldsymbol{B}|_{\max }$. This interpretation implies the resolution effect on the amplification factor. Actually the resolution dependence can be seen in some models with a weak initial field, and this is discussed later in the next section.

The time evolutions of the average field strength $|\boldsymbol{B}|_{\text {ave }}$ are also shown in Figure 5. Here the average is taken over the regions that the pressure is larger than $10 P_{0}$ in order to eliminate the preshocked regions. These time profiles of the maximum and average field are qualitatively quite similar to those in the realistic interstellar simulations [e.g., Fig. 5 in Inoue et al. (2012)]. This resemblance reminds that the RMI would be a fundamental process of the field amplification in the ISM. However, our results are still restricted within the two-dimensional picture. Obviously three-dimensional consideration will be an important next step for further understandings.

We found some curious features in the field evolution for the parallel shock case. Figures $7 a$ and $7 b$ show snapshots of the field lines and the size of the stretching term at $k v_{\text {lin }} t=6$. For this case, the position of the maximum field is quite different from that in the other cases. The field lines are concentrated at the stem of the mushroom rather than the interface or the mushroom cap. The heavy fluid " 2 " near the interface moves horizontally toward the $y$-axis just after the shock passage. The magnetic field is frozen into the fluid and thus it is gathered near the $y$-axis by this motion. The converging flow can escape toward the mushroom cap along the field lines so that it can be realized as incompressible motion. The largely amplified region coincides to where the stretching term, $B_{y}\left(\partial v_{y} / \partial y\right) \approx-B_{y}\left(\partial v_{x} / \partial x\right)$, is the highest. Interestingly, the magnetic field is amplified selectively in the fluid " 2 " for the parallel shock cases. For the perpendicular shock cases, the velocity pattern is the same, but the converging flow basically moves along the field lines and thus no field amplification at the stem part.

\section{DISCUSSIONS}

\subsection{Parameter Dependence}

In this section, we discuss the dependence of the field amplification on the model parameters such as the Mach number $M$, and the density jump $\rho_{2} / \rho_{1}$, and the initial field strength $\beta_{0}$. It is found that the amplification of a magnetic field by the RMI occurs in a variety of cases with a wide range of the initial parameters. The amplification factor is more than 100 for most of the cases. Therefore, this process should be a quite common phenomena, especially in astrophysical shock events with a high Mach number.

Figure 8 shows the evolutions of the maximum field strength for various models. The dependence on the shock strength is shown in Figure 8 8 . The Mach number ranging from $M=1.5$ to 100 are examined. The model parameters except for $M$ are identical to the fiducial model and the initial field orientation is in the $x$ direction. When $M \gtrsim 3$, the amplification factor exceeds $10^{3}$ in $k v_{\text {lin }} t=30$ as shown in the figure. The time history of $|\boldsymbol{B}|_{\max }$ in terms of the normalized time $k v_{\text {lin }} t$ looks quite similar for all the models. The am- 
plification process seems to be independent of the Mach number for this parameter range. This is because the stretching rate of the interface in the normalized unit has little dependence on the Mach number. Thus, even for the weak shock case with $M=1.5$, the magnetic field can be amplified by more than 100 times in $k v_{\text {lin }} t \sim 10$. These results are for the cases of the perpendicular shock, but the same conclusion can be obtained for the parallel shock cases. Notice that the growth velocity is nearly proportional to the Mach number. The normalization of the time is $t_{\mathrm{rm}}=1.17 t_{s 1}$ for the model with $M=1.5$ and $t_{\mathrm{rm}}=1.15 \times 10^{-2} t_{s 1}$ for $M=100$. The actual timescale of the RMI growth is largely different depending on the shock strength.

Figure $8 b$ demonstrates the dependence on the density jump at the interface. The models of the different density ratio, $\rho_{2} / \rho_{1}=100,10,3$, and 1.5 are shown in this figure. The other parameters and initial field geometry are the same as in the fiducial model. Again, the magnetic field is enhanced by more than two orders of magnitude in $k v_{\text {lin }} t \sim 10$ for all the models.

The amplification factor has a positive correlation with $\rho_{2} / \rho_{1}$. Since the growth velocity $v_{\text {lin }}$ has little dependence on $\rho_{2} / \rho_{1}$, the growth timescale is comparable for these cases; $t_{\mathrm{rm}}=0.40 t_{s 1}$ for $\rho_{2} / \rho_{1}=1.5$ and $t_{\mathrm{rm}}=0.19 t_{s 1}$ for $\rho_{2} / \rho_{1}=100$. However, the nonlinear behavior of the spike is found to be quite different at the later stage. The spike height reaches $y_{s} \approx 2.6 \lambda$ at $k v_{\text {lin }} t=30$ for the model with $\rho_{2} / \rho_{1}=100$, while it is only $y_{s} \approx 0.8 \lambda$ for $\rho_{2} / \rho_{1}=1.5$. When the density ratio $\rho_{2} / \rho_{1}$ is larger, the total magnetic flux swept by the spike is larger. This magnetic flux near the interface becomes a seed field to be stretched by the RMI. Then the maximum strength could be much higher as the ratio $\rho_{2} / \rho_{1}$ is larger. For the parallel shock cases, on the other hand, this trend can not be seen at all and the saturated amplitude is independent of $\rho_{2} / \rho_{1}$.

The time evolutions of the average field strength $|\boldsymbol{B}|_{\text {ave }}$ are also shown in Figure 8. The average is taken over the postshocked regions swept by the incident and transmitted shocks. For all the models, the maximum strength is much larger than the average. The strong field regions are highly localized along the stretched interface as a result of the RMI.

We performed some models that the ratio $\rho_{2} / \rho_{1}$ is less than unity. Although the phase of the RMI becomes reversed in those models, the magnetic field is amplified by many orders of magnitude in the same manner. It should be noted that the growth velocity given by Equation (8) is applicable for the reflected rarefaction wave. The phase inversion is expressed by the negative value of the growth velocity. The spike can penetrate deeply into the light fluid even for the rarefaction cases, and its structure does not change by much compared with that in the reflection shock cases. The efficient field amplification occurs mostly near the interface of the spike for the both cases.

\subsection{Saturation Level of Magnetic Field}

The dependence of the field amplification on the initial field strength is shown by Figure 9. The maximum strength $|\boldsymbol{B}|_{\max }$ in this figure is normalized by $\left(8 \pi P^{*}\right)^{1 / 2}$ where $P^{*}$ is the postshock pressure. The initial plasma beta of these models are $\beta_{0}=10^{8}, 10^{4}, 100$, and 1 .
The parameters except for $\beta_{0}$ are identical to the fiducial model and the magnetic field is initially aligned along the $x$-direction for all the models. When $\beta_{0}$ is large enough, the initial field is amplified more than two orders of magnitude. However, if $\beta_{0} \lesssim 100$, the amplification is limited; the factor is $\sim 10$ for $\beta_{0}=100$ and only a few for $\beta_{0}=1$.

When the initial strength of the ambient field becomes larger, the saturation level at the nonlinear regime is almost converged independent of $\beta_{0}$. The upper limit of the field strength is about $\beta^{*}=8 \pi P^{*} /|\boldsymbol{B}|_{\max }^{2} \sim 10$, which is roughly equal to the equipartition value to the thermal pressure after the shock heating. More precisely, the saturated magnetic field strength is determined by a balance with the kinetic energy of the growth velocity of the spike, $|\boldsymbol{B}|_{\max }^{2} / 8 \pi \approx \rho_{2}^{*} v_{\text {lin }}^{2} / 2$. For the strong shock cases, both of them can be comparable to the thermal energy. As seen from Figure $2 b$, the amplified field distributes along the interface at the mushroom cap with the thickness of $\sim 0.01 \lambda-0.02 \lambda$. If the magnetic pressure becomes comparable to the gas pressure, the interface stretching is reduced by the Lorentz force, and the increase of the maximum field strength will be saturated. Then the potential limit of the field amplification is determined by a condition that the RMI is suppressed by the amplified field itself.

In this figure, higher resolution results are also shown by the dotted curves. The same colors denote the same initial conditions. The resolution dependence can be seen for the models with a weaker initial field, where the higher resolution gives the higher saturation level. However, the upper limit of the saturated field has little dependence on the grid size. The parallel shock models shows qualitatively the same results for the dependence on the initial $\beta_{0}$ and the grid resolution. Therefore, we can conclude that the existence of the upper limit for the saturated field is a robust nature.

Even when the initial field strength is strong $\left(\beta_{0} \sim 1\right)$, the magnetic field can be amplified by a huge factor if the Mach number is much larger than about 10. For the strong shock cases, the postshock pressure $P^{*}$ is larger than $P_{0}$ by many orders of magnitude. Then the magnetic pressure of the initial field, $B_{0}^{2} / 8 \pi$, can be regarded as rather weak in terms of $P^{*}$. Therefore, the vorticity is deposited very close to the interface, and then the RMI can grow even in the presence of the strong field. As a result of the nonlinear growth of the RMI, the magnetic field is amplified up to the thermal value of the postshock pressure $P^{*}$.

The plasma beta in the ISM is usually close to unity (Beck 2001; Heiles \& Troland 2005). When $\beta_{0} \sim 1$ initially, the upper limit of the amplification factor is of the order of $|\boldsymbol{B}|_{\max } / B_{0} \sim\left(\beta_{0} P^{*} / P_{0}\right)^{1 / 2} \sim\left(P^{*} / P_{0}\right)^{1 / 2}$. If $P^{*} / P_{0} \gtrsim 10^{4}, 100$-fold enhancement of the magnetic field can be expected. The postshock pressure $P^{*}$ is determined just by the Mach number $M$ and density ratio $\rho_{2} / \rho_{1}$. The pressure ratio $P^{*} / P_{0}$ is nearly proportional to the square of the Mach number, but has little dependence on the density ratio. When the Mach number is larger than $M \gtrsim M_{\text {crit }} \approx 50$, the shock heating enhances the pressure by more than four orders of magnitude $P^{*} / P_{0} \gtrsim 10^{4}$. The Mach number of the shocks associated with SNRs is sufficiently larger than this crit- 
ical value. If the initial field strength is assumed to be about the average value of the ISM, $\sim 5 \mu \mathrm{G}$, then the enhanced maximum field reaches to the level of milligauss. Therefore, this mechanism could explain the origin of the strong field spots observed in the downstream regions of the front shock of SNRs (Uchiyama et al. 2007; Uchivama \& Aharonian 2008).

The critical Mach number $M_{\text {crit }} \approx 50$ is independent of the initial corrugation amplitude of the interface, but the size of $\psi_{0}$ affects the growth velocity and timescale of the RMI. Assuming $\psi_{0} / \lambda=0.1$, the growth time $t_{\mathrm{rm}}$ is much shorter than the sound crossing time, $t_{\mathrm{rm}} \lesssim 0.03 t_{s 1}$, when $M \gtrsim 50$. Since the cooling time is comparable to $t_{s 1}$, the magnetic field can be amplified significantly before the decrease of the pressure by the radiative cooling. The ionization fraction of the postshocked media is sufficiently large enough that the non-ideal MHD effects, such as the ambipolar diffusion and ohmic dissipation, are ignorable. Then the dissipation timescale of the amplified field is much longer than the RMI timescale.

In our numerical analysis, a discontinuous density jump is assumed at the interface. However, for the cases of the ISM, there must be a transition layer with a finite thickness between two different media. Then we also examined the effects of the transition layer on the field amplification. The density profile of the layer is approximately given by a function $\propto \tanh \left[\left(y-Y_{\mathrm{cd}}\right) / L_{t}\right]$ where $L_{t}$ denotes the thickness of transition layers. Although the growth rate of the magnetic field decreases with the increase of the thickness $L_{t}$, the saturation level of the maximum strength is found to be independent of $L_{t}$ if the transition layer is thinner than the corrugation wavelength $L_{t} \lesssim \lambda$. Thus the field amplification by the RMI could be realized even in the typical situations of the ISM.

\subsection{Observed Emissions and Particle Acceleration}

If the entire region of SNR RX J1713.7-3946 is magnetized up to $B \sim 1 \mathrm{mG}$, the origin of gamma-rays cannot be leptonic (Uchivama et al. 2007), which is inferred from the observed power ratio of the synchrotron to inverse-Compton emissions (Aharonian et al. 2007). However, when the strongly magnetized regions is caused by the RMI after the shock-cloud interaction, the averaged field strength of the overall SNR would be much smaller than milligauss, depending on the volume filling factor of the cloud in the upstream ISM. Therefore, the origin of gamma-ray from RX J1713.7-3946 should be discussed carefully taking account of other observational features, such as the spectrum of the gamma-ray emission (Abdo et al. 2011; Inoue et al. 2012) and structure of surrounding ISM (Ellison et al. 2010; Fukui et al. 2012).

As we estimated in $\S 2.2$, the timescale of field amplification by the RMI in young SNRs is about $100 \mathrm{yr}$, which indicates that the high-energy electrons accelerated at the forward shock (or the transmitted shock) by the diffusive shock acceleration are cooled down due to the synchrotron loss before the field strength reaches milligauss-order. Thus, in order to explain observed synchrotron X-ray hot spots, an additional accelerator other than the forward shock is necessary to produce brightening of the synchrotron X-rays at the region of $B \sim 1 \mathrm{mG}$. Inoue et al. (2012) discussed that the re- flected shock waves induced by the shock-cloud interactions can accelerate the electrons even in the downstream region of the forward shock, and which could explain the synchrotron emissions from strongly magnetized regions.

\section{SUMMARY}

We have investigated the evolution of a magnetic field associated with the RMI by using two-dimensional MHD simulations. In terms of the field amplification, the importance of "laminar stretching" driven by the RMI at the interface is successfully demonstrated. In our singlemode analysis, an incident shock propagating through a light fluid is considered to encounter a contact surface of a heavy fluid. When the interface is spatially corrugated, the RMI takes place and a mushroom-shaped structure develops in the density profile. An ambient magnetic field is initially supposed to be uniform and subthermal. Our numerical results for various situations suggest that the RMI is an efficient mechanism of the amplification of the interstellar magnetic fields. The main conclusions are summarized below.

1. The fluid motions associated with the RMI strengthen an ambient magnetic field by many orders of magnitude. This phenomenon can be seen in a wide range of the initial parameters. The amplification factor is almost independent from the Mach number of the incident shock and the initial field direction, so that it could occur even for the cases with a weak shock and/or a small density jump. Therefore we can conclude that the RMI is a robust mechanism of the ambient field amplification.

2. The physical mechanism of the field amplification is stretching associated with the nonlinear evolution of the RMI. The magnetic field is amplified efficiently at where the stretching term of the induction equation is predominant over the other terms. In most cases, the strong field regions are localized along the mushroom-shaped interface and form filamentary structures. Curiously, only for the parallel shock cases, the maximum field appears at the stem of the mushroom in the heavier fluid.

3. The amplified magnetic field is saturated when the magnetic pressure becomes comparable to the thermal pressure after the shock heating. This is because of the suppression of the RMI through the Lorentz force of the amplified magnetic field. If the Mach number of the incident shock is larger than about 50, we can expect at least more than 100-fold enhancement of the initial field. Thus the RMI can be a promising origin of the interstellar strong fields observed at the shock of SNRs (Uchivama et al. 2007).

The MHD RMI would play an important role not only in other astrophysical phenomena (e.g., Inoue et al. 2011) but also in many scientific fields such as interplanetary shocks $(\mathrm{Wu} 2003)$ and inertial confinement fusion (Lindl et al. 1992; Holmes et al. 1999). In this paper, the evolutions of an external magnetic field are examined. However, particularly for the case of laser plasmas, a selfgenerated field often cannot be ignorable. The baroclinic 
term generates a magnetic field as well as the vorticity. Then proper treatment of two-fluid effects should be included in the analysis for that purpose, and which will be an interesting subject for our future work.

The RMI has been studied extensively by laboratory experiments (e.g., Niederhaus \& Jacobs 2003; Chapman \& Jacobs 2006). Laser plasmas can be a new platform to examine the RMI in laboratories (Dimonte \& Remington 1993) and the inclusion of the effects of a magnetic field will be possible in such experiments (Kuramitsu et al. 2011). Therefore the magnetic field amplification proposed in this paper could be tested by laser experiments in the near future.

We thank Shu-ichiro Inutsuka, Tomoyuki Hanawa, and Takahiro Kudoh for useful discussions and comments. Computations were carried out on SX-8R at the Cybermedia Center and SX-9/B at the Institute of Laser Engineering of Osaka University.

\section{APPENDIX}

\section{VORTEX SHEET MODEL}

Using the circulation $\Gamma=\phi_{1}-\phi_{2}$ and average velocity potential $\Phi$ defined by $\Phi=\left(\phi_{1}+\phi_{2}\right) / 2$, we rewrite the Bernoulli equation (13) as

$$
\frac{D \Gamma}{D t}=2 A^{*} \frac{D \Phi}{D t}-A^{*} \boldsymbol{q} \cdot \boldsymbol{q}+\frac{A^{*}+2 \tilde{\alpha}}{4} \boldsymbol{\kappa} \cdot \boldsymbol{\kappa}-\tilde{\alpha} A^{*} \boldsymbol{\kappa} \cdot \boldsymbol{q},
$$

in which the derivative $D / D t$ is given by

$$
\frac{D}{D t}=\frac{\partial}{\partial t}+\overline{\boldsymbol{v}} \cdot \nabla, \quad \overline{\boldsymbol{v}}=\boldsymbol{q}+\frac{\tilde{\alpha} \boldsymbol{\kappa}}{2},
$$

where $\boldsymbol{q}=\nabla \Phi, \boldsymbol{\kappa}=\nabla \Gamma, A^{*}$ is the Atwood number defined by $A^{*}=\left(\rho_{1}^{*}-\rho_{2}^{*}\right) /\left(\rho_{1}^{*}+\rho_{2}^{*}\right)$, and $\tilde{\alpha}=\tilde{\alpha}\left(A^{*}\right)(|\tilde{\alpha}| \leq 1)$ is a weighting factor such that $\tilde{\alpha} \neq 0$ for $A^{*} \neq 0$ (Matsuoka \& Nishihara 2006). Here, the spatial derivative is taken at the interface.

We regard the interface in the RMI as a curve in the $x$ - $y$ plane, and parameterize it using a Lagrangian parameter $\theta$. The velocity of the interface $(x, y)=(X(\theta, t), Y(\theta, t))$ is derived as

$$
X_{t}=U+\frac{\tilde{\alpha} X_{\theta}}{2 s_{\theta}} \kappa, \quad Y_{t}=V+\frac{\tilde{\alpha} Y_{\theta}}{2 s_{\theta}} \kappa
$$

(Baker et al. 1982; Matsuoka \& Nishihara 2006), where the vortex sheet strength $\kappa$ is defined by $\kappa=\partial \Gamma / \partial s=\Gamma_{\theta} / s_{\theta}, s$ is arc length of the sheet, and the subscript denotes the differentiation with respect to the variable. The vortex-induced velocity $U=U(\theta, t)$ and $V=V(\theta, t)$ are given by the Birkhoff-Rott equation:

$$
\begin{gathered}
U(\theta, t)=-\frac{1}{4 \pi} \int_{-\pi}^{\pi} \frac{\sinh \left[Y(\theta, t)-Y\left(\theta^{\prime}, t\right)\right] \kappa\left(\theta^{\prime}, t\right) s_{\theta}\left(\theta^{\prime}\right) d \theta^{\prime}}{\cosh \left[Y(\theta, t)-Y\left(\theta^{\prime}, t\right)\right]-\cos \left[X(\theta, t)-X\left(\theta^{\prime}, t\right)\right]+\delta^{2}}, \\
V(\theta, t)=\frac{1}{4 \pi} \int_{-\pi}^{\pi} \frac{\sin \left[X(\theta, t)-X\left(\theta^{\prime}, t\right)\right] \kappa\left(\theta^{\prime}, t\right) s_{\theta}\left(\theta^{\prime}\right) d \theta^{\prime}}{\cosh \left[Y(\theta, t)-Y\left(\theta^{\prime}, t\right)\right]-\cos \left[X(\theta, t)-X\left(\theta^{\prime}, t\right)\right]+\delta^{2}}
\end{gathered}
$$

(Birkhoff 1962; Rott 1956), where we regularize the Cauchy integral using Krasny's $\delta$ (Krasny 1987).

Differentiating Equation (A1) with respect to $\theta$, we obtain the following Fredholm integral equation of the second kind:

$$
\kappa_{t}=\frac{2 A^{*}}{s_{\theta}}\left(X_{\theta} U_{t}+Y_{\theta} V_{t}\right)-\frac{\left(1+\tilde{\alpha} A^{*}\right) \kappa}{s_{\theta}^{2}}\left(x_{\theta} U_{\theta}+y_{\theta} V_{\theta}\right)+\frac{A^{*}+\tilde{\alpha}}{4 s_{\theta}}\left(\kappa^{2}\right)_{\theta} .
$$

Solving Equations (A2) and (A5) simultaneously, we can determine the motion of a vortex sheet in the RMI.

For the fiducial model, the fluctuation amplitude after shock passed is approximately calculated as $\psi_{0}^{*} / \lambda=0.06188$. The density for the both layers are $\rho_{1}^{*} / \rho_{1}=6.716$ and $\rho_{2}^{*} / \rho_{1}=39.549$, so that the Atwood number is $A^{*}=-0.7097$ for this case. The initial condition in Figure $4 d$ is then given by

$$
X(\theta, 0)=\theta, \quad Y(\theta, 0)=a_{0} \cos \theta, \quad \kappa(\theta, 0)=-\frac{2 \sin \theta}{s_{\theta}(0)},
$$

where the initial amplitude $a_{0}$ is set to $a_{0}=2 \pi \psi_{0}^{*} / \lambda=0.3888$.

In the numerical calculation, the stretching rate, i.e., the rate of the temporal change of length $s(\theta)$ at the Lagrangian point $\theta$ is defined by

$$
\frac{1}{\bar{s}(\theta)} \frac{d s(\theta)}{d t}=\frac{1}{\bar{s}(\theta)} \frac{s(\theta, t+\Delta t)-s(\theta, t)}{\Delta t},
$$

where $\bar{s}=[s(t+\Delta t)+s(t)] / 2$ and the length $s(\theta)$ is calculated by $\int s_{\theta} d \theta$, in which the integral is performed as the spectral integration with respect to $\theta$. Here, we set the time step $\Delta t=0.0002$, the regularized parameter $\delta=0.15$, and the weighting factor $\tilde{\alpha}=-0.05$. We adopt the trapezoidal rule and the forth-order Runge-Kutta method for the 
spatial and temporal integration, respectively. For detailed numerical schemes, refer to Matsuoka \& Nishihara (2006) and references therein.

\section{REFERENCES}

Abdo, A. A., et al. 2011, ApJ, 734, L27

Aharonian, F., et al. 2007, A\&A, 464, 235

Armstrong, J. W., Rickett, B. J., \& Spangler, S. R. 1995, ApJ, 443, 209

Baker, G. R., Meiron, D. I., \& Orszag, S. A. 1982, J. Fluid Mech., 123,477

Bamba, A., Yamazaki, R., Ueno, M., \& Koyama, K. 2003, ApJ, 589,827

Beck, R. 2001, Space Sci. Rev., 99, 243

Bell, A. R. 1978, MNRAS, 182,147

Berezhko, E. G., Ksenofontov, L. T., \& Völk, H. J. 2003, A\&A, 412, L11

Birkhoff, G. 1962, in Hydrodynamic Instability: Proceedings of the 13th Symposium in Applied Mathematics, ed. G. Birkhoff, R. Bellman, \& C. C. Lin (Providence: American Mathematical Society), 55

Blandford, R. D., \& Ostriker, J. P. 1978, ApJ, 221, L29

Brouillette, M. 2002, Annu. Rev. Fluid Mech., 34, 445

Bykov, A. M., Uvarov, Y. A., \& Ellison, D. C. 2008, ApJ, 689, L133

Cao, J., Wu, Z., Ren, H., \& Li, D. 2008, Phys. Plasmas, 15, 042102

Chapman, P. R., \& Jacobs, J. W. 2006, Phys. Fluids, 18, 074101

Chevalier, R. A. 1992, Nature, 355, 617

Clarke, D. A. 1996, ApJ, 457, 291

Colella, P., \& Woodward, P. R. 1984, J. Comp. Phys., 54, 174

Dimonte, G. \& Remington, B. 1993, Phys. Rev. Lett., 70, 1806

Ellison, D. C., Patnaude, D. J., Slane, P., \& Raymond, J. 2010, ApJ, 712, 287

Endeve, E., Cardall, C. Y., Budiardja, R. D., \& Mezzacappa, A. 2010, ApJ, 713, 1219

Evans, C. R., \& Hawley, J. F. 1988, ApJ, 332, 659

Field, G. B. 1965, ApJ, 142, 531

Field, G. B., Goldsmith, D. W., \& Habing, H. J. 1969, ApJ, 155, L149

Fukui, Y., et al. 2012, ApJ, 746, 82

Giacalone, J., \& Jokipii, J. R. 2007, ApJ, 663, L41

Guo, F., Li, S., Li, H., Giacalone, J., Jokipii, J. R., \& Li, D. 2012 ApJ, 747, 98

Hanawa, T., Mikami, H., \& Matsumoto, T. 2008, J. Comp. Phys., 227,7952

Heiles, C., \& Troland, T. H. 2005, ApJ, 624, 773

Holmes, R. L., et al. 1999, J. Fluid Mech., 389, 55

Inoue, T., Asano, K., \& Ioka, K. 2011, ApJ, 734, 77

Inoue, T., Inutsuka, S., \& Koyama, H. 2006, ApJ, 652, 1331
Inoue, T., Yamazaki, R., \& Inutsuka, S. 2009, ApJ, 695, 825

Inoue, T., Yamazaki, R., Inutsuka, S., \& Fukui, Y. 2012, ApJ, 744,71

Ishizaki, R., \& Nishihara, K. 1997, Phys. Rev. Lett., 78, 1920

Koyama, H., \& Inutsuka, S. 2000, ApJ, 532, 980

-. 2002, ApJ, 564, L97

Krasny, R. 1987, J. Fluid Mech., 184, 123

Kuramitsu, Y., et al. 2011, Ap\&SS, 336, 269

Lindl, J. D., McCrory, R. L., \& Campbell, E. M. 1992, Phys. Today, 45, 32

Long, K. S., Reynolds, S. P., Raymond, J. C., Winkler, P. F., Dyer, K. K., \& Petre, R. 2003, ApJ, 586, 1162

Matsuoka, C., \& Nishihara, K. 2006, Phys. Rev. E, 73, 026304

Matsuoka, C., Nishihara, K., \& Fukuda, Y. 2003, Phys. Rev. E, 67, 036301

Meshkov, E. E. 1969, Fluid Dyn., 4, 101

Mizuno, Y., Pohl, M., Niemiec, J., Zhang, B., Nishikawa, K.-I., \& Hardee, P. E. 2011, ApJ, 726, 62

Niederhaus, C. E. \& Jacobs, J. W. 2003, J. Fluid Mech., 485, 243

Nishihara, K., Wouchuk, J. G., Matsuoka, C., Ishizaki, R., \& Zhakhovsky, V. V. 2010, Phil. Trans. R. Soc. A, 368, 1769

Richtmyer, R. D. 1960, Commun. Pure Appl. Math., 13, 297

Rott, N. 1956, J. Fluid Mech., 1, 111

Samtaney, R. 2003, Phys. Fluids, 15, L53

Sano, T., Inutsuka, S., \& Miyama, S. M. 1998, ApJ, 506, L57

Shin, M.-S., Stone, J. M., \& Snyder, G. F. 2008, ApJ, 680, 336

Stone, J. M., \& Norman, M. L. 1992, ApJS, 80, 791

Stone, J. M. \& Zweibel, E. G. 2009, ApJ, 696, 233

Uchiyama, Y., \& Aharonian, F. A. 2008, ApJ, 677, L105

Uchiyama, Y., Aharonian, F. A., Tanaka, T., Takahashi, T., \& Maeda, Y. 2007, Nature, 449, 576

van Leer, B. 1979, J. Comp. Phys., 32, 101

van Loo, S., Falle, S. A. E. G., Hartquist, T. W., \& Moore, T. J. T. 2007, A\&A, 471, 213

Völk, H. J., Berezhko, E. G., \& Ksenofontov, L. T. 2005, A\&A, 433,229

Wheatley, V., Pullin, D. I., \& Samtaney, R. 2005, Phys. Rev. Lett., 95, 125002

Wheatley, V., Samtaney, R., \& Pullin, D. I. 2009, Phys. Fluids, 21,082102

Wouchuk, J. G., \& Nishihara, K. 1996, Phys. Plasmas, 3, 3761 —. 1997, Phys. Plasmas, 4, 1028

Wu, C. C. 2003, Space Sci. Rev., 107, 219

Wu, C. C., \& Roberts, P. H. 1999, Geophys. Res. Lett., 26, 655 

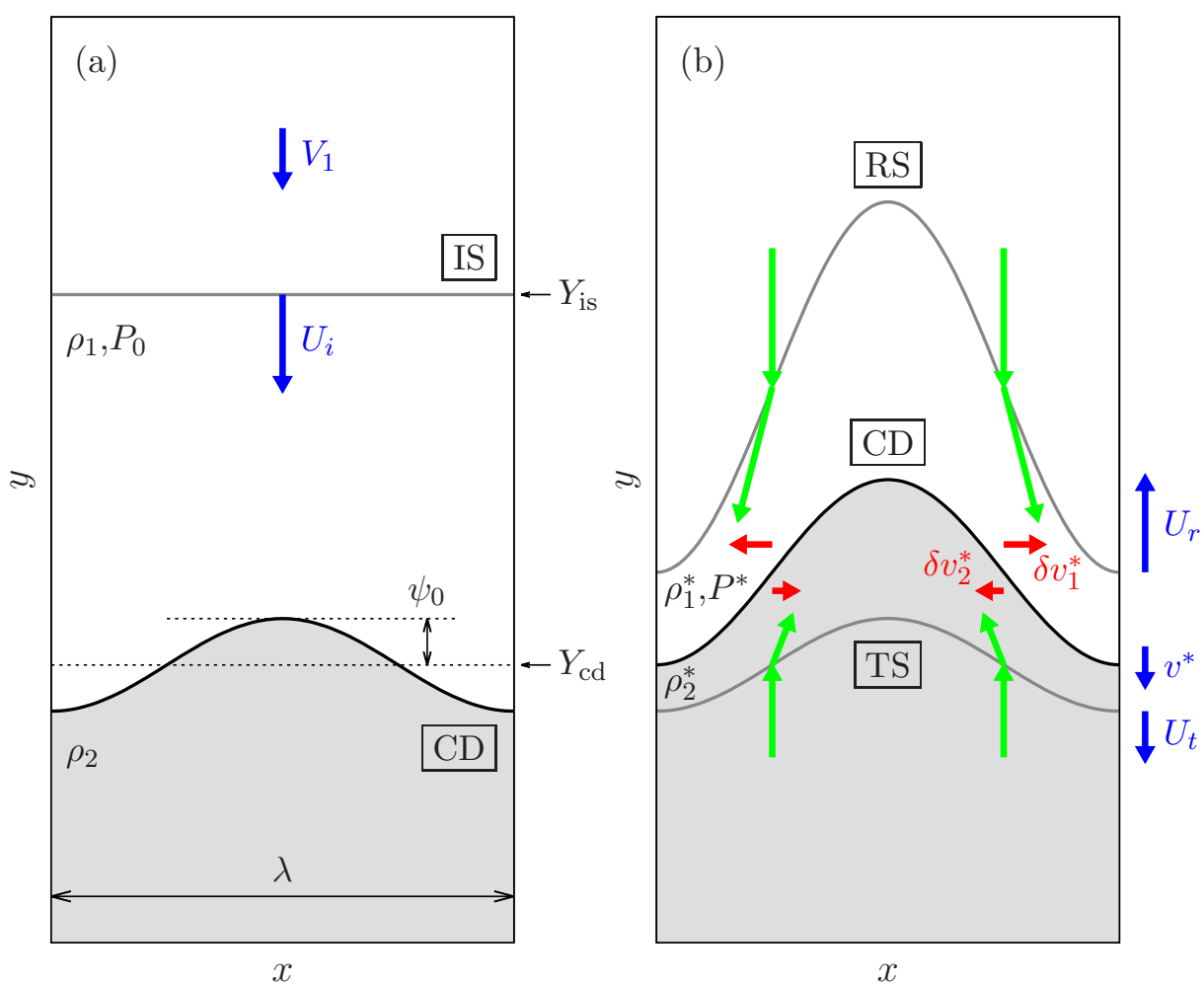

FIG. 1. - (a) Schematic picture of the initial configuration for single-mode analysis of the RMI. Two fluids are divided by a contact discontinuity (CD). The densities of the light fluid " 1 " and heavy fluid " 2 " are $\rho_{1}$ and $\rho_{2}$, and the uniform pressure for the both fluids is $P_{0}$. The interface is corrugated sinusoidally with the wavelength $\lambda$ and the amplitude $\psi_{0}$. An incident shock (IS) propagates in the light fluid " 1 " with the shock velocity $U_{i}$. The shock strikes the corrugated interface at a time $t=0$. Here $V_{1}$ is the flow velocity behind the incident shock. (b) Sketch of the shock-front shapes after the incident shock hits the corrugated interface. The transmitted shock (TS) and reflected shock (RS) travel from the contact discontinuity in the opposite direction with the velocities $U_{t}$ and $U_{r}$, respectively. The pressure and velocity at the contact discontinuity are $P^{*}$ and $v^{*}$, and the densities behind the transmitted and reflected shocks are $\rho_{1}^{*}$ and $\rho_{2}^{*}$. Because of the obliqueness of the shock surface, tangential flows, $\delta v_{1}^{*}$ and $\delta v_{2}^{*}$, are generated at the both side of the interface. Refraction of the fluid motions at the transmitted and reflected shocks are shown by the thick arrows. 

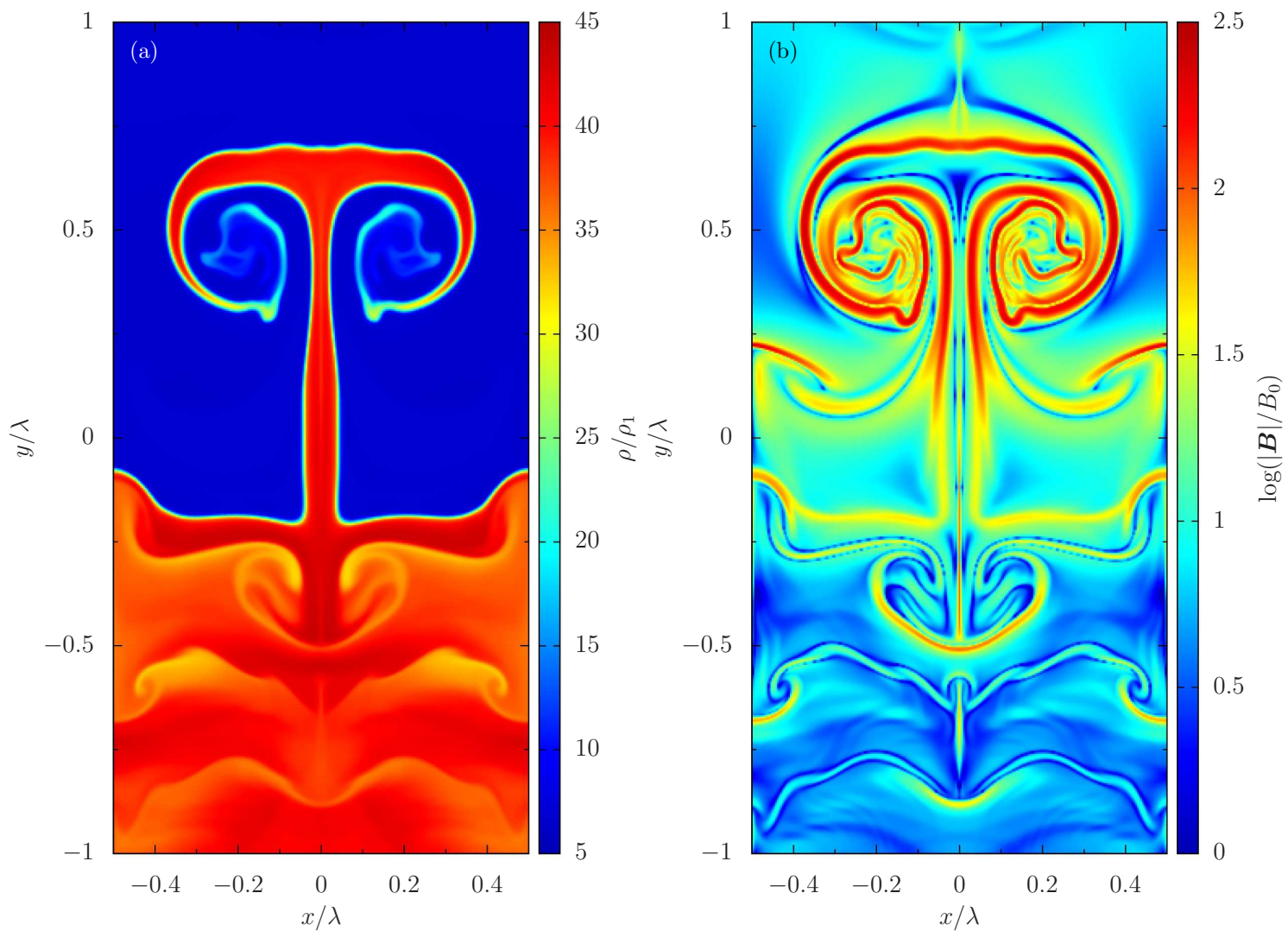

FIG. 2. - Spatial distributions of (a) the density and (b) the magnetic field strength at a nonlinear stage of the RMI for the fiducial model. The model parameters are $M=10, \rho_{2} / \rho_{1}=10, \psi_{0} / \lambda=0.1$, and $\beta_{0}=10^{8}$. The direction of the initial ambient field is in the $x$-direction, or perpendicular to the incident shock velocity (perpendicular MHD shock). These snapshots are taken at the normalized time $k v_{\text {lin }} t=10$. The maximum field strength at this time is $|\boldsymbol{B}| \max / B_{0}=272$.
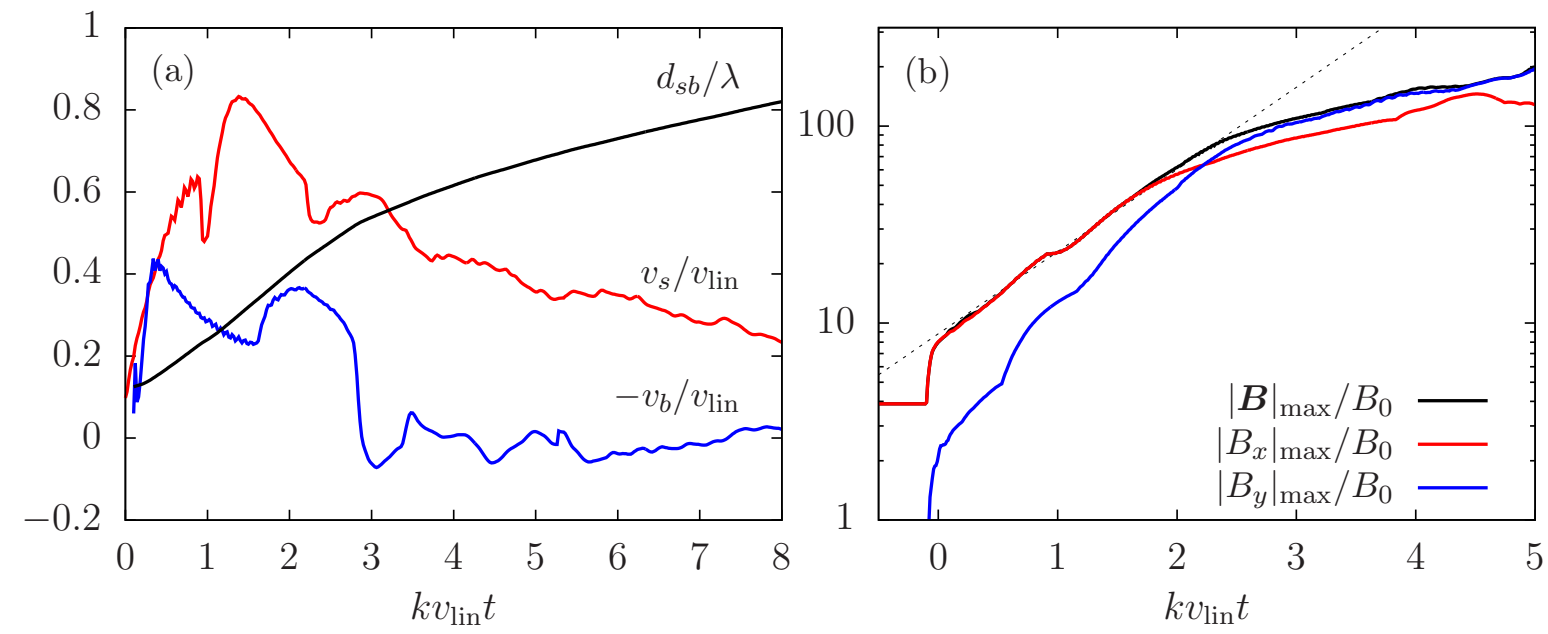

FIG. 3.- (a) Evolution of the growth velocities of the spike $v_{s}$ and the bubble $v_{b}$, and the distance from the spike top to the bubble bottom $d_{s b}$ in the fiducial model, which are normalized by the asymptotic linear growth velocity $v_{\text {lin }}$ and the wavelength of the fluctuation $\lambda$. (b) Time profile of the maximum of the magnetic field strength $|\boldsymbol{B}| \max$ in the fiducial model. The maximum values of each component, $\left|B_{x}\right|_{\max }$ and $\left|B_{y}\right|_{\max }$, are also shown. The exponential growth at the early stage until $k v_{\text {lin }} t=2$ can be fitted by a function $\propto \exp \left(\sigma_{B} t / t_{\mathrm{rm}}\right)$ with $\sigma_{B}=1.0$ where $t_{\mathrm{rm}}=\left(k v_{\mathrm{lin}}\right)^{-1}$ is the characteristic timescale of the RMI. 

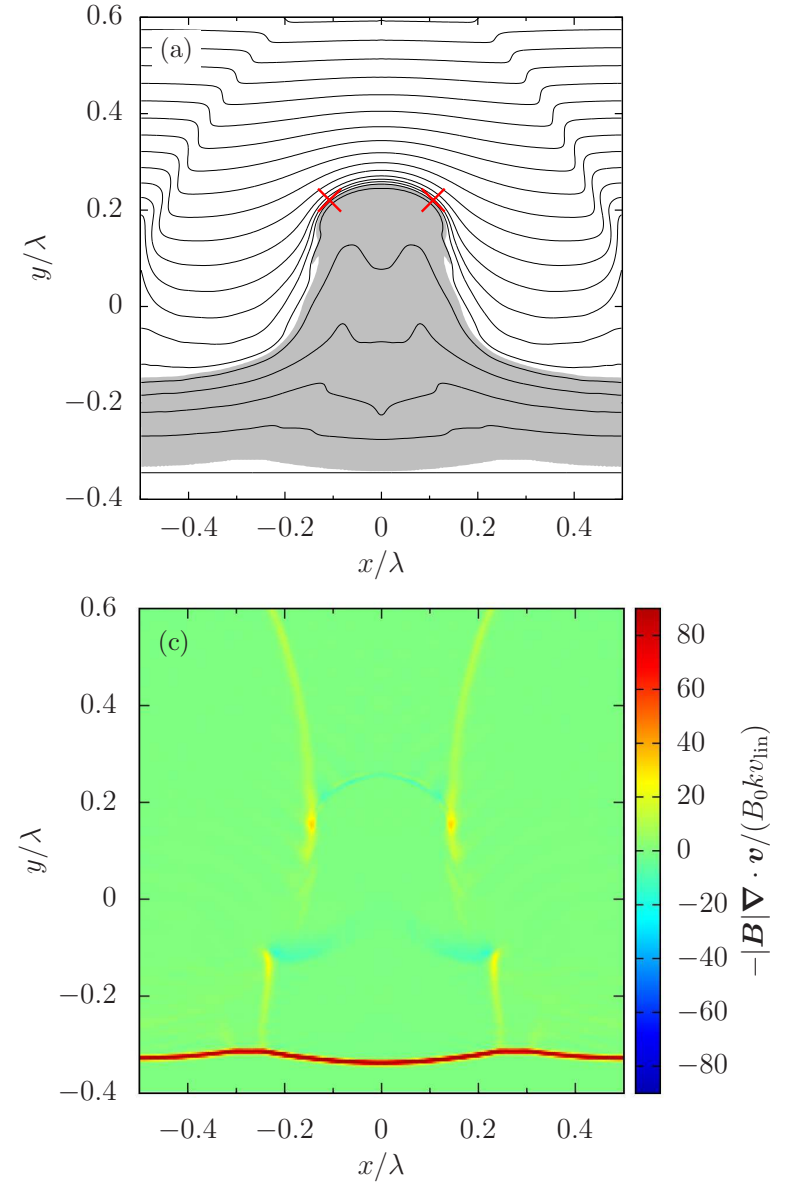
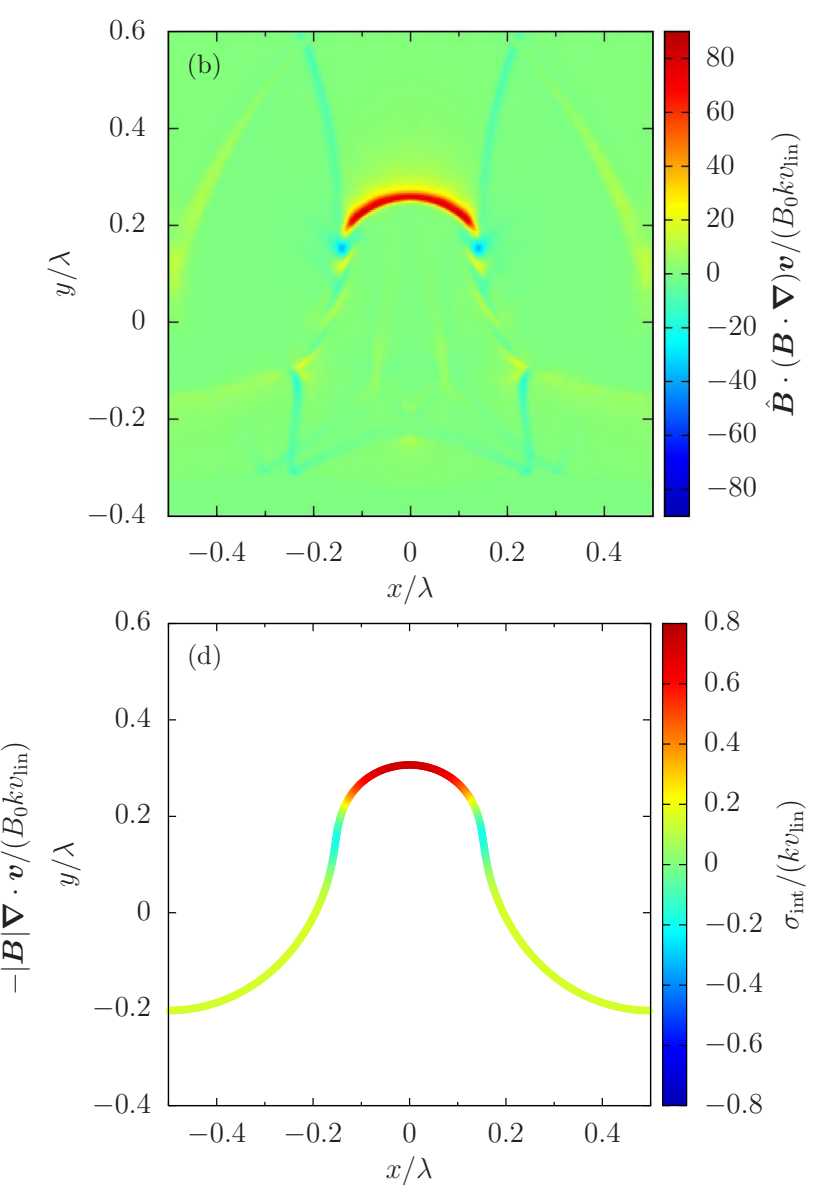

Fig. 4.- (a) Magnetic field lines for the fiducial model at $k v_{\operatorname{lin}} t=2$. The gray color denotes the higher density regions in the fluid "2" compressed by the transmitted shock. The positions of the maximum field are shown by the crosses. (b,c) Relative importance of (b) "stretching" and (c) "compression" in the induction equation (12) calculated from snapshot data of the fiducial model at $k v_{\text {lin }} t=2$. Each term is normalized by a constant $B_{0} k v_{\text {lin }}$ and $\hat{\boldsymbol{B}} \equiv \boldsymbol{B} /|\boldsymbol{B}|$ is a unit vector. The same color bars are used for these figures. (d) Interface profile predicted by a vortex sheet model. The model parameters corresponding to the fiducial model are used for the numerical calculation. The line color indicates the stretching rate of the interface $\sigma_{\text {int }}$, i.e., the rate of the temporal change of length at each Lagrangian point.

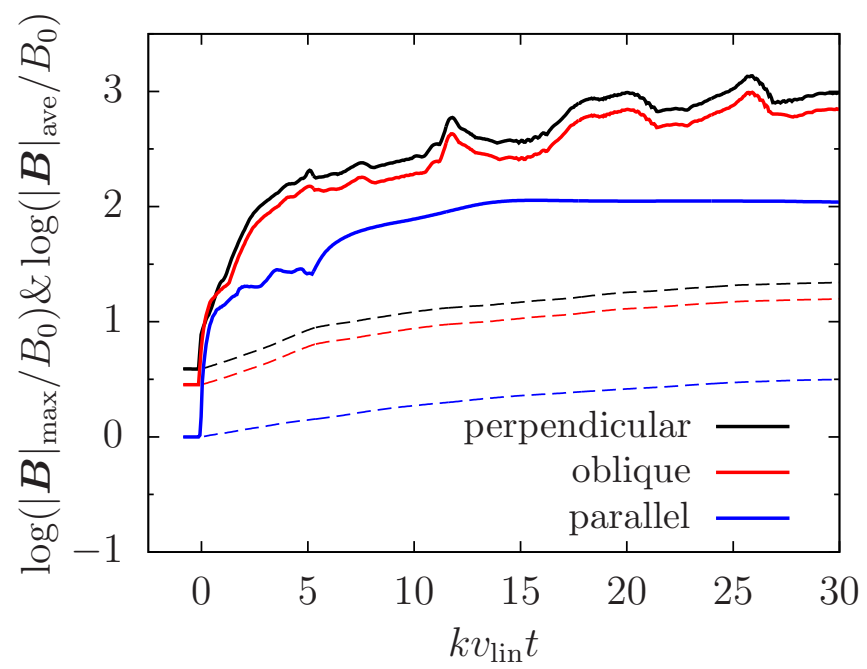

FIG. 5. - Time evolution of the maximum field strength $|\boldsymbol{B}|_{\max }$ shown as a function of the normalized time $k v_{\text {lin }} t$ for the cases with different orientations of the initial field. In the perpendicular shock case (fiducial model), the initial field is $\left(B_{x}, B_{y}\right)=\left(B_{0}, 0\right)$. For the parallel shock case and the oblique shock case, the uniform field direction is assumed to be $\left(B_{x}, B_{y}\right)=\left(0, B_{0}\right)$ and $\left(B_{x}, B_{y}\right)=$ $\left(B_{0} / \sqrt{2}, B_{0} / \sqrt{2}\right)$, respectively. The other parameters are the same as in the fiducial model. The thin dashed curves are time evolution of the average field strength $|\boldsymbol{B}|_{\text {ave }}$ for each case. The average is taken over only the postshocked regions. 

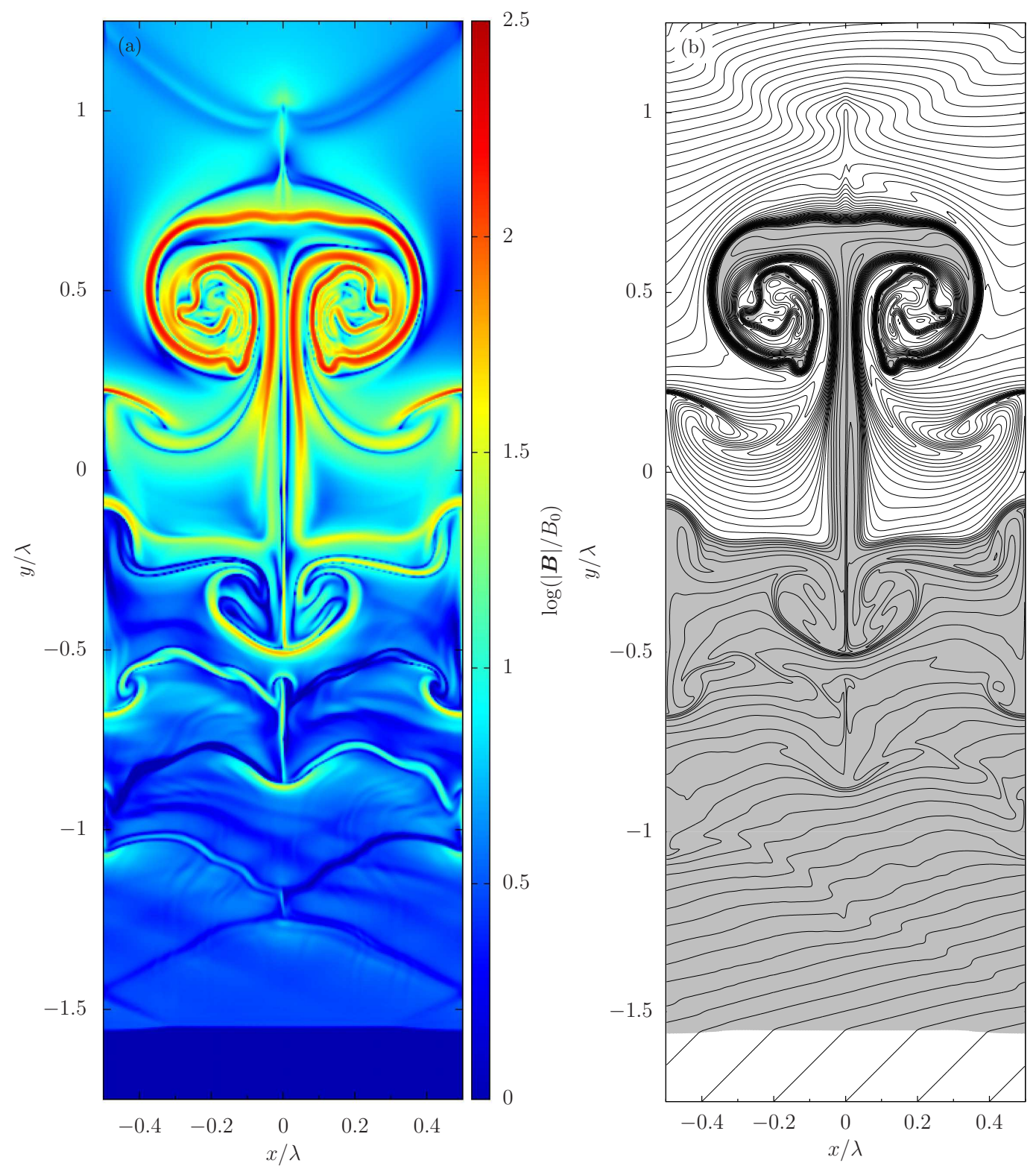

FIG. 6. - Spatial distributions of (a) the magnetic field strength and (b) the field lines for the oblique shock case. The direction of the initial ambient field is 45 -degree to the $x$-axis. The other parameters are identical to those in the fiducial model. These snapshots are taken at the normalized time $k v_{\text {lin }} t=10$. The higher density regions bounded between the contact discontinuity and the transmitted shock front are depicted by the gray color in (b). 

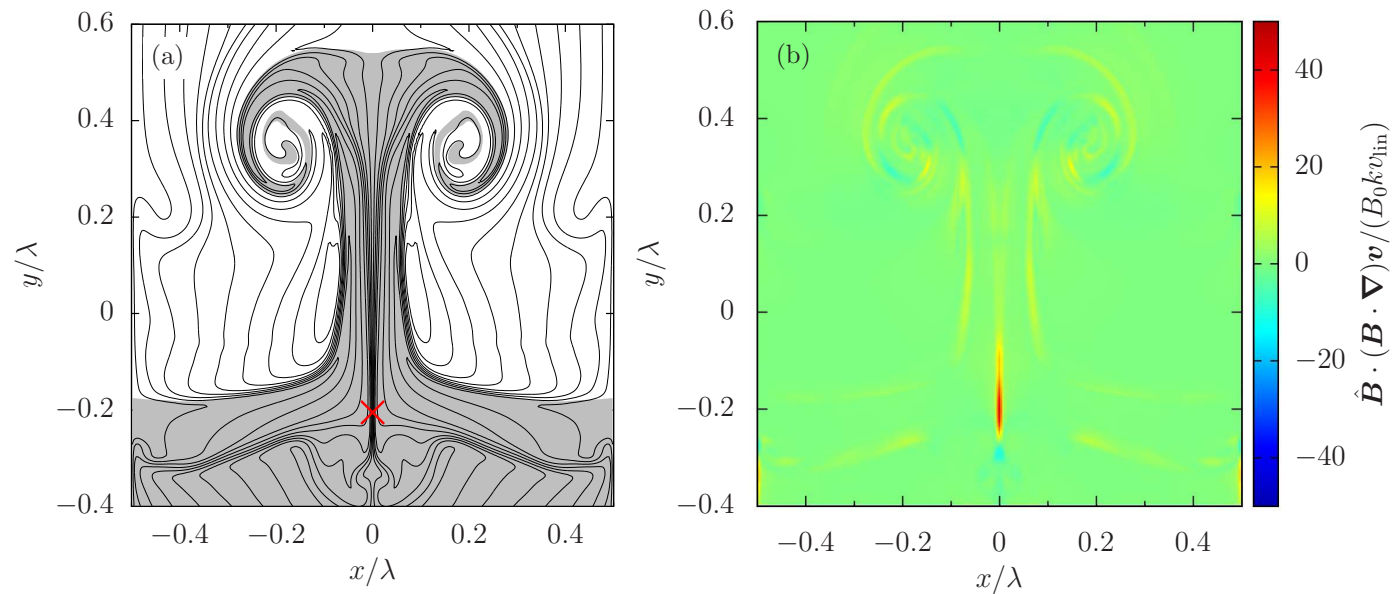

Fig. 7. - (a) Magnetic field lines of the parallel MHD shock case at $k v_{\text {lin }} t=6$. The model parameters are identical to the fiducial model, but the initial field direction is parallel to the incident shock velocity for this case. The gray color denotes the higher density regions in the fluid "2" compressed by the transmitted shock. The position of the maximum field is shown by the cross mark. (b) Distribution of the size of "stretching" term in the induction equation (12) calculated from the snapshot data same as in Figure $7 \mathrm{~h}$. The stretching term is normalized by the same constant $B_{0} k v_{\text {lin }}$ as in Figure 4 .
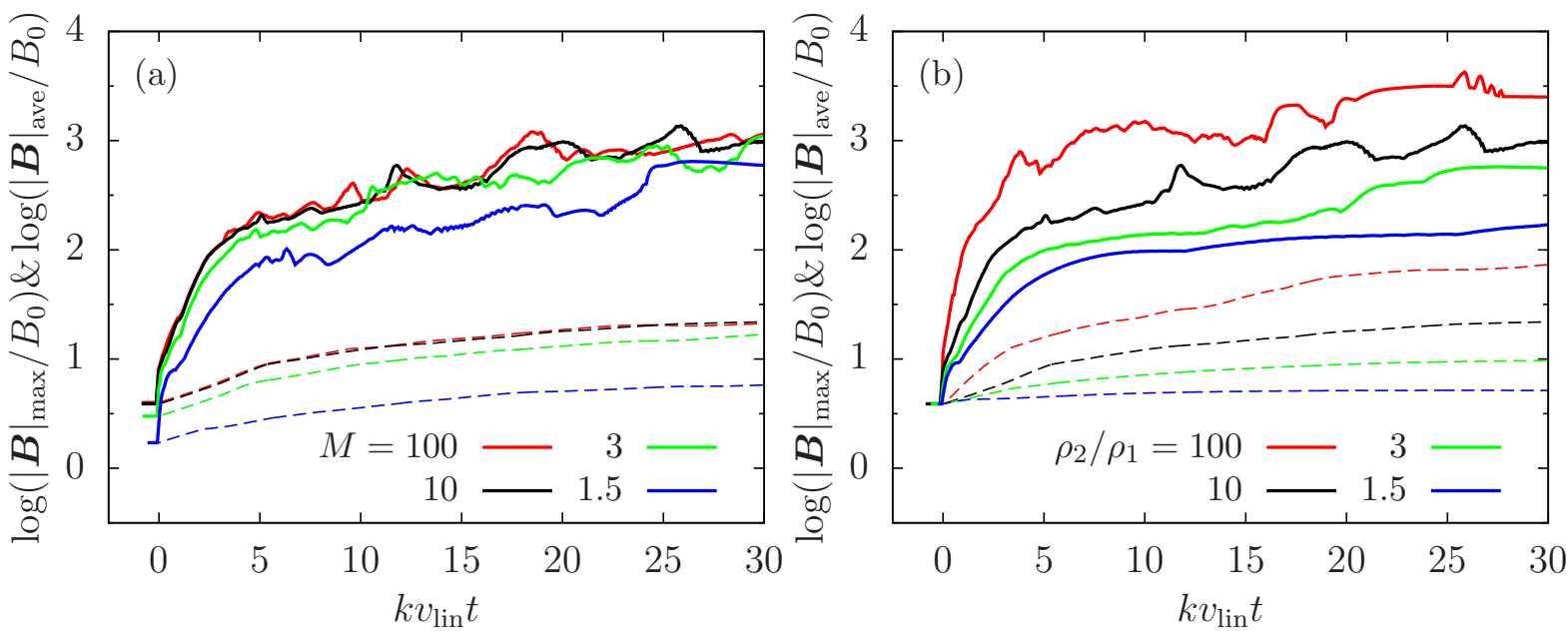

Fig. 8.- (a) Dependence of the maximum field strength on the Mach number $M$. The time profiles of $|\boldsymbol{B}| \max$ are shown as a function of the normalized time $k v_{\text {lin }} t$ for the models with different shock strength; $M=100, M=10$ (fiducial model), $M=3$, and $M=1.5$. The model parameters except for $M$ are identical to those of the fiducial model. The thin curves are time evolution of the average field strength $|\boldsymbol{B}|_{\text {ave }}$ for each case. The average is taken over only the postshocked regions. (b) Dependence of the maximum field strength on the density jump $\rho_{2} / \rho_{1}$. The time evolution of $|\boldsymbol{B}|_{\max }$ are drawn for the models with different density ratio; $\rho_{2} / \rho_{1}=100, \rho_{2} / \rho_{1}=10$ (fiducial model), $\rho_{2} / \rho_{1}=3$, and $\rho_{2} / \rho_{1}=1.5$. Other than $\rho_{2} / \rho_{1}$, the same parameters as in the fiducial model are used. The thin curves are time evolution of the average field strength $|\boldsymbol{B}|_{\text {ave }}$ for each case. 


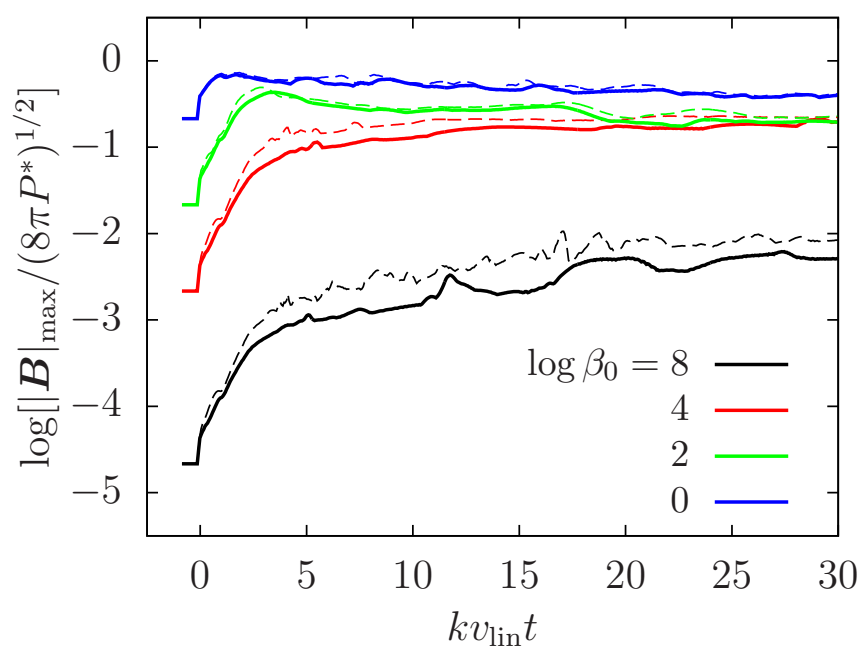

FIG. 9. - Dependence of the saturation level of the amplified magnetic field on the initial plasma beta $\beta_{0}=8 \pi P_{0} / B_{0}^{2}$. The maximum field strength $|\boldsymbol{B}|_{\max }$ is normalized by $\left(8 \pi P^{*}\right)^{1 / 2}$ using the postshock pressure $P^{*}$. The model parameters are the same as in the fiducial model except for the initial field strength $B_{0}$, which is indicated by the line colors; $\beta_{0}=10^{8}$ (fiducial model), $\beta_{0}=10^{4}, \beta_{0}=10^{2}$, and $\beta_{0}=1$. The initial field direction is assumed to be in the $x$-direction for all the models. For the purpose of comparison, higher resolution results are also shown in this figure by the thin dashed curves. The grid size of the higher resolution runs is $\Delta_{x}=\Delta_{y}=\lambda / 512$ which is twice as good as the standard resolution. 